\title{
Memory and cognitive effects of ECT: informing and assessing patients ${ }^{\dagger}$
}

\author{
Harold Robertson \& Robin Pryor
}

\begin{abstract}
Over the past few years electroconvulsive therapy (ECT) has come under increased scrutiny in the UK, with the first systematic review of patients' experiences and new national guidelines. Our aim in this article is to translate recent and sometimes confusing research and policy statements into practical guidance that benefits patients. We examined the evidence on the permanent memory and cognitive effects of ECT, with a focus on delineating their nature, understanding how ECT may cause them, informing prospective patients about them, and assessing their impact on former patients. We describe a simple and effective method for assessing retrograde amnesia. Data do not exist at this time to confirm the mechanisms by which ECT exerts its adverse effects, but clinicians should fully inform patients of the possible permanent adverse effects of the treatment, which include amnesia, memory disability and cognitive disability, and should provide follow-up testing using relevant instruments.
\end{abstract}

InJanuary 2002, as part of a review of electroconvulsive therapy (ECT) undertaken by the UK's Department of Health, the Service User Research Enterprise (SURE) published the first-ever systematic review of patients' views on ECT (Service User Research Institute, 2002). The review encompassed several large-scale surveys by or of people who had received ECT in the UK (United Kingdom Advocacy Network, 1996; ECT Anonymous, 1999; Pedler, 2000). In April 2003, the National Institute for Clinical Excellence (now the National Institute for Health and Clinical Excellence, NICE) issued guidance on the use of ECT, and at the same time, the UK ECT Review Group published a review of its safety and efficacy (National Institute for Health and Clinical Excellence, 2003; UK ECT Review Group, 2003). The Royal College of Psychiatrists has established the ECT Accreditation Service and revised its guidelines for practitioners to take into account the NICE advice (Royal College of Psychiatrists, 2005).

Some of the conclusions to come out of the new work-in particular, that at least one-third of patients experience permanent amnesia (Service User Research Institute, 2002; Rose et al, 2003; Scott, 2005), that half of patients had not received an adequate explanation prior to treatment (Rose et al, 2003,

${ }^{\dagger}$ For a commentary on this article see pp. 237-238, this issue.
2005; Philpot et al, 2004) and that newer methods of ECT have not resulted in an appreciable decrease in adverse effects (UK ECT Review Group, 2003) - suggest that changes are overdue in both practice and policy.

The new evidence presents opportunities for improving clinical care in several areas: delineating the nature of ECT's permanent adverse effects; developing adequate and relevant tools to assess patients; and providing consent that is fully informed.

\section{Defining deficits}

It is evident from a close reading of patient reports such as those documented by SURE that 'memory' is too simple a term to encompass the range of ECT's permanent adverse effects, yet there has been almost no work done on improving terminology (Box 1). The confusion goes back to the first instrument specifically designed to assess people given ECT, the Squire Memory Questionnaire (SMQ; Squire et $a l$, 1979). The SMQ was developed to distinguish between the cognitive impairments associated with depression and those caused by ECT. Although Squire and his colleagues believed that they had done this (Squire \& Slater, 1983; Squire \& Zouzounis, 1988), others have not used the test for its intended

Harold Robertson (148 Beach 94th St, Suite 6, 3rd Fl., Queens, NY 11693, USA. Email: robertson.harold@gmail.com) is the director of a not-for-profit charitable foundation in New York focusing on the underserved patient population. His interest in psychiatric research, in particular research into the use of electroconvulsive therapy, has brought him into contact with others with similar interests across the globe for the past 20 years. Robin Pryor is a psychologist who has held a private practice in New York, NY, for 30 years. His particular interests are the history of psychiatry and first-person narratives of mental health service users. He writes and lectures on these topics. 


\section{Box 1 Terminology for ECT's adverse effects}

Memory

- Autobiographical memory: one's store of knowledge of past experiences and learning

- Amnesia: the loss of autobiographical memory, i.e. the erasure of all that was thought, done and learned during a particular period. Amnesia is different from 'forgetting' in that the memories and knowledge are obliterated and cannot be accessed by effort or reminders

- Retrograde amnesia: amnesia for the period prior to ECT

- Anterograde amnesia: amnesia for the period after ECT (see also memory disability below)

- Working memory: one's ability to store and access information in everyday life

- Memory disability (often called anterograde amnesia): the loss of working memory

\section{Cognition}

- Higher mental functions, including attention, concentration, flexibility, problemsolving, analytical and abstract thinking, reasoning, executive function, intelligence

- Cognitive disability: the loss of any of the higher mental functions of cognition

purpose, and it is hard to say whether the SMQ has muddied the water more than it has cleared it. Although often spoken of as if it measured a unitary entity 'memory', the SMQ actually encompasses multiple dimensions of cognition: attention, alertness, concentration, learning. It does not at all address the most common effect of ECT, which is variously called amnesia, retrograde amnesia or memory loss. By these terms is generally understood the obliteration of a specific time period in a person's life.

It is when 'memory' is used as a shorthand term for both retrograde amnesia and ongoing difficulties with memory function in the present that confusion ensues, a confusion that intensifies when the latter is sometimes called 'anterograde amnesia'. Although most will understand anterograde amnesia to mean ongoing memory disability, this is not always the case. The US National Institute of Mental Health has defined anterograde amnesia as the inability to remember events that happened after ECT (National Institutes of Health, 1985) and the College's new handbook (Royal College of Psychiatrists, 2005) appears to use it in the same way.

To confuse matters further, the term 'short-term memory loss' is sometimes used as a synonym for anterograde amnesia. Short term to some will mean temporary, to others it will be seen as a description of the type of memory that is affected - the ability to retain information for a short period, or working memory (Baddeley \& Hitch, 1974) - which says nothing about its longevity. The term 'temporary' rather than 'short-term' should always be used to refer to effects that resolve, but even then it should be used with caution because neuropsychology recognises that transient impairment of cognitive function may have residual permanent effects. 'Dysfunction' or 'disability' should be used rather than 'loss' (which implies a one-time event) to refer to ongoing difficulties with memory ability and cognition.

If the term anterograde amnesia must be used, it should be clearly defined as difficulties with memory in daily life, and examples given (Box 2).

Inevitably, memory overlaps with and subsumes other cognitive functions, such as learning and attention as well as overall intelligence. When individuals who have had ECT report ongoing memory disability, it is necessary for a clinician trained in neuropsychological evaluation to tease out the roles played by attention, concentration, overall slowed mental processing and deficits of executive function such as inability to shift mental set. The ECT psychiatrist and treatment team may not be trained in neuropsychological evaluation, since outside of research settings it is not routinely performed on people who have had ECT. When it is, it is usually initiated by the patient, not the doctor. Because of this, the treating psychiatrist may fear personal liability and thus be unwilling to attribute deficits to ECT.

It has long been known that ECT can produce deficits in non-memory-related cognitive function (Calev, 1994). However, long-term studies comparing controls and people who have had ECT to determine when and if non-memory cognitive function normalises after ECT have not been done. A comprehensive battery of neuropsychological tests carried out on individuals who had had ECT

\section{Box 2 Notes describing an individual's} memory disability (anterograde amnesia)

- Can't hold onto information

- Memory not as good as before or as good as that of others the same age

- Forgets where she put items such as keys

- Has to make lists all the time

- Can't remember faces and names

- Forgets what she was about to say or do

(after Freeman et al, 1980) 
between 9 months and 30 years previously revealed impairment on a range of measures, even after controlling for the effects of illness and medication (Freeman et al, 1980).

Despite recommendations that psychiatrists inform patients of non-memory cognitive aftereffects (Calev, 1994) and warn them that 'they are not going to function well on more tasks than they anticipate' (Calev et al, 1995), patients are still routinely not informed about these effects; there is no mention of them in the recommended consent forms of the American Psychiatric Association (APA; 2001), the Royal College of Psychiatrists (2005: Appendix IV) or the manufacturers of ECT equipment. This may contribute to the consistent findings (Rose et al, 2003, 2005; Philpot et al, 2004) that half of people given ECT say they did not receive an adequate explanation of the treatment.

The current APA consent forms not only contain no warnings about adverse effects on cognition, but advise that 'Most patients report that memory is actually improved by ECT' (American Psychiatric Association, 2001). This statement is contradicted by all service-user research as well as the findings of SURE (2002) and NICE (2003); indeed, Scott (2005) remarked that NICE took 'special note of the evidence from users that cognitive impairment after ECT often outweighed their perception of any benefit from it'.

\section{Is it depression?}

If the task of assessing amnesia, memory disability and cognitive deficits is left to a patient's treating psychiatrist, there may be a tendency to attribute all deficits, without evaluation, to depression, even when the patient has fully recovered. The APA guidelines state that

'Patients with the greatest symptomatic benefit from ECT typically report the greatest improvement in subjective evaluations of memory. Thus, when patients report subjective memory impairment after ECT, their mood as well as their cognition should be assessed' (American Psychiatric Association, 2001: p. 72).

It seems that this statement is based on SMQ scores from six studies: Pettinati \& Rosenberg, 1984; Weiner et al, 1986; Mattes et al, 1990; Sackeim et al, 1993 and Coleman et al, 1996, which involved the same patients; and Sackeim et al, 2000. On average, patients reported improvement in cognitive functions assessed by the SMQ within 1 week of ECT. However, the following should be taken into account: first, the improvement was relative only to immediate pre-ECT status, not baseline, thus in fact reflecting a net impairment; and second, objective testing revealed that the patients were in fact cognitively impaired post-ECT. There are other studies in which patients reported impairment postECT on the SMQ (Squire et al, 1979; Squire \& Slater, 1983; Squire \& Zouzounis, 1988). To the extent that a handful of studies support a claim of correlation between memory and cognitive self-rating and mood during or immediately after ECT, there might be a correlation between relatively improved memory self-rating and improved mood. There is no evidence of a correlation between impaired memory/cognition after ECT and impaired mood, much less a causal relationship.

\section{The problem of premature assessment}

There are many reasons why hospitalised patients who have received ECT might overestimate their abilities. After each treatment they experience acute organic brain syndrome (Sackeim, 1986). In hospital, they are not exposed to even minimally taxing actions such as shopping and driving. There are no environmental cues as to what they are expected to know and remember in their roles outside the hospital. In a few days or even weeks, patients cannot gain enough experience of using their minds and memories to accurately assess their altered capacities (Weiner et al, 1986; Coleman et al, 1996; Donahue, 2000). In the longer term, i.e. 2-6 months, patients who initially rated their memory and cognition as improved, experience and accurately report impairment (Weiner et al, 1986; Coleman et al, 1996).

More recent work using the SMQ suggests that, in the short term as well, patient ratings of memory function are negative and are correlated with the results of objective tests, even when controlling for the level of depression. These researchers say that patient reports of memory impairment 'must not be dismissed as being depressive complaints only' (Schulze-Rauschenbach et al, 2005).

\section{Differentiating the effects of ECT}

Although terms such as memory loss are often used interchangeably by clinicians to describe the temporary effects of depression on cognition (especially attention) and the long-lasting effects of ECT on a range of cognitive functions, this confusion is unnecessary and could be avoided. The effects of ECT are quantitatively and qualitatively different from those of depression (Squire et al, 1979) and researchers have consistently distinguished between them (Cronholm \& Ottoson, 1963; Squire et al, 1979; Squire \& Slater, 1983; Pettinati \& Rosenberg, 1984; Squire \& Zouzounis, 1988). Numerous controlled studies show that individuals who are depressed but have not had ECT do not suffer amnesia (Janis, 1950; 
Weiner et al, 1986). People who have experienced the effects of both depression and ECT rarely mistake one for the other (Food and Drug Administration, 1982; Donahue, 2000): ECT's effects are different and worse, they occur only after ECT and they persist in the absence of depression and drugs.

\section{Possible mechanisms of action}

How might ECT cause permanent amnesia and memory and cognitive disability? There are several theories (Box 3). One is that memory is affected because the applied electrical current is densest in the medial temporal area structures associated with memory, including the hippocampus; these areas have low seizure thresholds. However, this has not been studied directly (Calev, 1994).

Other theories focus on ECT's effects on brain metabolism and neurochemistry: breach of the blood-brain barrier and increased cerebral blood pressure (Bolwig et al, 1977; Taylor et al, 1985); regional increases in T2 relaxation times (Diehl et $a l, 1994)$; disturbance of the long-term potentiation mechanism (Sackeim, 2000; Rami-Gonzalez et al, 2001); excessive release of excitatory amino acids and activation of their receptors (Chamberlin \& Tsai, 1998; Rami-Gonzalez et al, 2001), and decreased cholinergic transmission (Khan et al, 1993; RamiGonzalez et al, 2001). Even temporary alterations in any of these may have permanent effects on the brain.

Since ECT affects both temporal and frontal lobes, it is logical that its effects would not be limited to amnesia, but would involve both memory and non-memory neuropsychological functions (Calev et al, 1995). Sackeim (2000) hypothesises that the traditional view that amnesia results from damage to medial temporal lobe structures alone may be wrong, since it is known both that frontal lobe damage can result in amnesia as extensive as that

\section{Box 3 Possible causes of ECT's adverse} effects

- Direct effects of electricity on the hippocampus

- Breach of the blood-brain barrier

- Increase in cerebral blood pressure

- Regional increases in T2 relaxation times

- Disturbance of the long-term potentiation mechanism

- Excessive release of excitatory amino acids and activation of their receptors

- Decreased cholinergic transmission seen after ECT and that ECT exerts its most profound effects on the prefrontal cortex.

If this hypothesis holds, then frontal functions must be affected as well as memory. Simply because there has been very little investigation of ECT's effects on these functions, doctors should not be sanguine as to lack of permanent effects. Absence of evidence is not evidence of absence. In particular, Sackeim (2000) points to the lack of formal research on ECT's effects on the executive functions of the prefrontal cortex: working memory (holding onto information in the service of a range of cognitive functions), logical reasoning and abstraction, shifting of mental set, problem-solving, planning and organising. These are 'fundamental to organising one's life and controlling behavior, yet there has been little investigation of the impact of ECT' (Sackeim, 2000).

Three trials, two controlled and one small and uncontrolled, support the theory of frontal lobe involvement in functional impairment, although assessments were carried out only during or immediately after ECT (Neylan et al, 2001; RamiGonzalez et al, 2003; Schulze-Rauschenbach et al, 2005).

A generation ago, one researcher, reviewing the literature on ECT experimentation, wrote that the ease of its administration has resulted in its widespread use

\begin{abstract}
'without the usual background information customarily thought appropriate for most treatment modalities ... this is undoubtedly the case because of the clinically observed changes in affect and behavior that result from such treatment. While such behavioral observations are certainly fruitful, such a model should be reversed to allow behavioral inferences to the possible effects on neocortical structures of such a procedure' (Goldstein et al, 1977).
\end{abstract}

\section{The evidence base}

In the absence of long-term follow-up studies over the past two decades, the best available evidence for the permanent effects of ECT on memory ability and cognition has been generated by former patients. This has most often taken the form of patient-designed survey instruments, which ask specifically about cognition. Of the groups whose findings were incorporated into the SURE systematic review, one found that $65 \%$ of people who had had ECT reported impaired organisational skills (ECT Anonymous, 1999). Another found that one-third had difficulty concentrating, and $15 \%$ reported loss of reasoning ability (Pedler, 2001). A third asked people whether they had experienced a loss of intelligence 'soon after the treatment', and about $40 \%$ answered affirmatively (they were not asked whether the loss persisted) (Philpot et al, 2004). 
However, former patients have publicly testified that $\mathrm{ECT}$ can result in a very significant ( $>30$ point) permanent decrement in IQ score (Food and Drug Administration, 1982; Andre, 2001; Cott, 2005: p. 5 ) and have documented the claims by extensive neuropsychological evaluation.

Although surveys and case reports are not rigorous controlled trials, in the absence of such trials conducted months or years after ECT, they provide a basis for inferences as to the treatment's permanent adverse effects and possible mechanisms of action.

\section{What's wrong with the way patients have been assessed?}

Claims that ECT does not have permanent adverse effects on memory and cognitive ability have been based on extremely gross measures of mental function such as the Mini-Mental State Examination (MMSE; Folstein et al, 1975) and other dementia screening scales (Stoudemire et al, 1993; Sackeim, 2000; McCall et al, 2004). But if ECT had produced wholesale dementia on a scale gross enough to be detected by these tests, it would have been abandoned decades ago.

Researchers have used very simple, brief measures to assess patients - typically, highly structured tests of verbal learning involving familiar material. Examples include the Auditory Verbal Learning Test (AVLT; Rey, 1964) and various forms of paired associates, with very short retention intervals. But there is no evidence that ECT interferes with wellestablished skills such as vocabulary or with short recall periods (Squire \& Chace, 1975; Zervas \& Jandorf, 1993).

Even people with severe brain injury or lobotomy can perform well on simple tests of overlearned verbal material that require culturally common information, for example the Wechsler Memory Scale. Highly motivated and concerned ECT patients are even more likely to do well on these tests. However, clinicians who conclude from this that there is 'no memory loss' have not measured memory loss at all, and certainly not the type of memory and cognitive disability that people can experience after ECT (National Insitute for Clinical Excellence, 2003).

Collectively, the comments of people who have had ECT indicate loss of complex skills that underlie real-world roles such as student, professor, nurse or physicist, and often inability to return to those roles post-ECT (Box 4).

The sensitivity of the tests used after ECT depends largely on whether and how well they reflect actual cognitive demands of the type placed on ex-patients. Researchers have assured patients that ECT has
Box 4 A representative sample of patient reports of permanent amnesia and disability

'I've got 13 GCEs, top grade, but no professional qualifications since ECT. I've sat only one exam, and despite its being $70 \%$ project work and continual assessment, I've struggled to just pass, well bottom - my memory and impaired concentration can't cope.'

'After ECT I could no longer play my guitar. I could not remember chord sequences/ patterns, words or songs that I had performed hundreds of times before ECT. The ability to play or learn new music has never returned.'

'In addition to destruction of entire blocks of pre-ECT memories, I have continued to have considerable difficulty in memory recall with regard to academic pursuits. I have been forced to tape-record all education materials that require memorisation. I was forced to "re-take" accounting. Now I am again forced to "re-take" a basic one semester course in computerised word processing.'

'I can't remember new information with the ease that I could before ECT. Distractions and interruptions seriously interfere with information retention, and any new bit of information may "cancel out" the bit that preceded it.'

'I have trouble with my memory today. My IQ was 120 before the treatments and it is not anywhere near that now. I have trouble just trying to cook a meal. I do not work. I make lists so that I can try to recall what I need to do.'

'I had to drop out of school when I realised I could not remember what I had studied before entering the hospital, and I was totally unable to absorb new material. I continue to have difficulty concentrating for extended periods of time.'

'Before ECT, I studied math up through calculus. After ECT, I can just barely make change in a store. ECT gives a person a different brain from the one a person had.'

(Food and Drug Administration, 1982; Pedler, 2001; Service User Research Enterprise, 2002)

no permanent adverse effects on the basis of the assumption that these demands will be minimal (McCall et al, 2004). But this assumption has never been tested, and patient reports warn against it. The 
ECT patient population includes people who are in the prime of life, highly educated and involved in demanding professions, and who can be very articulate in describing their deficits. If simple standardised tests cannot detect these deficits, the challenge is not to dismiss their comments but to find or devise more appropriate tests.

\section{The Autobiographical Memory Interview}

Weiner et al (1986) and Sackeim and his colleagues (Coleman et al, 1996; Sackeim et al, 2000) have attempted to measure amnesia with an unvalidated instrument of their own design, known as the (Columbia University) Autobiographical Memory Interview. This test is insensitive to ECT-induced amnesia in two related ways: it measures very old information whereas ECT amnesia is known to be densest for more recent memory; and as many as $60 \%$ of the 200-300 test items involve overlearned and highly rehearsed facts - grandparents' names, telephone numbers of close relatives, etc. - which are not likely to be erased by ECT. The overlearned and the old information may overlap (as in questions such as 'What is your address') or it may not, but in either case confounding the testing with these factors will unnecessarily result in an underestimate of the extent of retrograde amnesia.

Furthermore, the Autobiographical Memory Interview assumes that amnesia is limited to events that took place within the 12 months prior to ECT and does not attempt to assess amnesia that is not limited to that time period. However, only about $20 \%$ of the questions ask specifically about that year; the rest ask about overlearned personal information (What are your parents' names? What are the rooms in your house?) or about events that have 'ever' happened to patients or their families.

Thus, it is remarkable that even as insensitive an instrument as this has shown extensive permanent retrograde amnesia measured at 2 months (Coleman et al, 1996) and 6 months (Weiner et al, 1986) after ECT.

\section{Assessment of amnesia}

Routine neuropsychological tests are unhelpful in attempting to assess retrograde amnesia (Rose et al, 2003). Squire \& Slater (1983) attempted to measure amnesia by asking people who had had ECT to make a time line showing the amount of life lost. The accuracy of this depends, of course, on the patients' ability to assess the extent of their amnesia, which can take many years, as they can only discover what they have forgotten when prompted by others to remember it. If asked soon after ECT, they are very likely to underestimate the extent of retrograde amnesia.

\section{Box 5 Neuropsychological batteries suitable for use after ECT}

Non-verbal and visuospatial memory and reasoning

- Benton Visual Retention Test (Sivan, 1992)

- Bender Gestalt (Bender, 1938)

- Test of Non-verbal Intelligence (Brown et al, 1982)

Working memory

- Digits Backwards (Wechsler, 1997)

- Speaking Span Test (Daneman \& Green, 1986)

Executive function

- Wisconsin Card Sort (Heaton, 1981)

- Halstead Category (Reitan \& Wolfson, 1993)

- Booklet Category (de Filippis et al, 1979)

Reasoning and working memory

- Sub-tests of the Wechsler (1997) such as Arithmetic and Picture arrangement

Because the information stored in memory is unique to each individual, standardised questionnaires or checklists may prove insensitive to amnesia even when the patient can describe or demonstrate it. Janis (1950) interviewed patients before and 1 month after ECT. He suggested general topics but let the patients speak at length. After ECT, he attempted to elicit the same information, but could not. For each individual he could count 10-20 significant life experiences that had been erased that were not limited to the period immediately before the treatment. Even when he prompted patients to recount events they had described in great detail before treatment, they could neither recall nor recognise them. One year after ECT, the amnesias remained stable. The same interviews were given to controls matched in all ways: age, gender, education, duration of hospitalisation, type and duration of mental disorder; the controls, who had not had ECT, had no amnesia.

The Janis test (Janis, 1950) can be done easily and cost-effectively even by those with no special research training. SURE (2002) in particular calls for the replication of this study, as have others over the years. But it has not yet been done.

\section{Assessment of memory and cognitive ability}

Tests of memory and cognitive ability must assess a range of functions, because ECT impairment may vary not only between individuals (Goldstein et al, 1977) but within individuals when they undergo 
more than one course of treatment. Simple tests of rote verbal learning or the memory sub-tests of the Wechsler are not sufficient, since ECT (if the amnesia is not catastrophic) spares vocabulary, overall wealth of knowledge and overlearned verbal skills. Patients who have had ECT should be evaluated with the type of neuropsychological batteries that would be used for patients with a known or suspected history of brain injury. These should include tests of nonverbal and visuospatial memory and reasoning such as those listed in Box 5.

If there are constraints of time and finances, tests should be tailored to the individual's deficits, which can be identified by narrative self-report and by rating scales such as the Cognitive Failures Questionnaire (Broadbent et al, 1982).

\section{When should patients be tested?}

If there is to be baseline testing, compensation must be made to account for the difference between the patient's true memory and cognitive capacity and the performance when preoccupied by depression, medicated or hospitalised. If such compensation is not made, all a return to 'baseline' function after ECT would show would be that ECT's effects are roughly equal to - although not necessarily the same as - those of depression (Calev et al, 1995).

A better estimation of pre-ECT capacity would be the patient's history and normal functioning at school, work or in some other capacity. Many patients, at least in the USA, will have had an IQ test, which can be used for comparison with postECT scores.

Patients cannot be meaningfully evaluated in hospital during or soon after ECT. Neither selfreports nor crude memory tests may be reliable (Cronholm \& Ottoson, 1963). A patient may do well on the MMSE or counting serial sevens but may not know that her friend visited her the day before - and will not know she doesn't know. Having had no reason theretofore not to trust her memory, and not having been warned to expect severe dysfunction, she will adamantly insist that her memory cannot be faulty. It is not the psychological defence mechanism of denial, nor is it only the acute organic brain syndrome which occurs with ECT, that causes this genuine unawareness. Most patients have never before experienced a day in their life when they did not know what they ate for dinner or who they had seen or what they had read the day before. They do not even know that this is possible, let alone that it is happening to them.

The ECT Accreditation Service (2005) recommends that patients should be interviewed 3 and 6 months after ECT. But at 3 months, they may not have recovered the ability to hold on to day-to-day memories (they may still be within the period of anterograde amnesia, estimated by the US National Institutes of Health (1985) to average 2 months). We propose that follow-up should be no sooner than 6 months. One year allows for optimal stabilisation of permanent cognitive deficits and better assessment of retrograde amnesia.

The Service User Research Enterprise (2002) has called for a research study with 'long followup because losses of memory prior to ECT may only become apparent after a long interval', as have Greenhalgh et al (2005: p. 78).

\section{What should patients be told?}

\section{Amnesia}

The clinician who tells her patients that there is a lack of research on the permanent adverse effects of ECT will certainly be on solid ground; however, this is unlikely to help patients in making a potentially life-altering decision. The best she can do is present her patients with what is known (and not known) and encourage them to assess the risk in light of their personal situation.

Thus, patients can be told that permanent amnesia is one of the 'common' (Sackeim, 2000) or 'serious / frequently occurring' (Royal College of Psychiatrists, 2005: p. 207) effects of ECT and that it affects at least one-third of patients (Service User Research Institute, 2002; Rose et al, 2003). Such amnesia may be presented as having multiple dimensions: the amount of life lost, the temporal gradient, the nature of what is lost, and the effect of the memory erasure on the individual's life.

The amount of life lost to amnesia cannot be predicted; patients should be warned that it has been known to extend to 10-20 years (Pedler, 2001; Service User Research Enterprise, 2002). It should be made clear that amnesia is not limited to information about discrete events or to facts that are easily regained, such as dates and telephone numbers, but that it encompasses all thoughts, feelings, personal interactions and relationships, learning and skills associated with the erased time period, and thus there is no simple or easy way to recapture what is lost. Since the temporal gradient of ECT amnesia is the opposite of normal forgetting, patients should be warned that the most recent months or years will be most affected.

When amnesia is permanent it has profound, rarely positive, effects on all aspects of the patient's subsequent life. For many people the effects of permanent amnesia and/or memory and cognitive disability negate any benefit sustained from ECT (National Institute for Clinical Excellence, 2003). 
The College now advises psychiatrists to discuss the topic of retrograde amnesia carefully (Royal College of Psychiatrists, 2005: p. 7). But profound and sudden retrograde amnesia has no parallel in ordinary human experience. Doctors cannot be expected to understand the myriad ways in which permanent amnesia can disrupt one's life. For this reason, prospective patients should be encouraged to speak with, or read accounts written by, people who have experienced amnesia. Such accounts are contained within the above-mentioned reports of SURE, NICE and MIND (Pedler, 2001), and are widely available in print (e.g. Donahue, 2000) and through online forums, e.g. http://www. ect.org) where prospective patients and families can sometimes ask questions directly of former patients.

\section{Cognitive impairment}

The Royal College of Psychiatrists (2005: p. 19) and NICE (2003) advise that the potential for cognitive impairment be highlighted during the consent process. Patients should be clearly told that ECT may have serious and permanent effects on both memory ability and non-memory cognition. These are best described in everyday terms: 'the ability to plan and organise and get things done' rather than 'executive function'.

Intact memory and intelligence are highly prized in our culture. The more valuable a possession, the more important it is to know about even a small chance that it might be permanently lost. Even if the answer to how often IQ is permanently lowered is 'We don't know', that is a material fact to be weighed by the patient. As individuals, patients vary greatly in the demands placed on their intellect and the potential consequences of permanent impairment. The decision to agree to ECT is theirs; the duty to inform, their physician's.

\section{Conclusions}

Evaluation and re-evaluation of ECT's risks and benefits by SURE, NICE and the Royal College of Psychiatrists, and the growing recognition of the extent and importance of research by and involving people who have experienced ECT, as well as increased interest in qualitative data, should lead to improvement in both patient care and research. In light of alarming findings that $50 \%$ of patients report receiving inadequate warnings of the potential sideeffects of ECT, informed consent practices need to be revised. In particular, prospective patients should be warned of the significant risk of permanent amnesia and the possibility of permanent memory and cognitive disability. Research to adequately assess the nature and longevity of these effects should be undertaken, 'incorporating patients' perspectives on the impact of ECT into future RCTs' (Greenhalgh et al, 2005: p. 78). By all accounts this is long overdue.

\section{Declaration of interest}

None.

\section{References}

American Psychiatric Association Committee on Electroconvulsive Therapy (2001) The Practice of Electroconvulsive Therapy: Recommendations for Treatment, Training, and Privileging. Washington, DC: American Psychiatric Association.

Andre, L. (2001) Testimony at the Public Hearing of the New York State (U.S.) Assembly Standing Committee on Mental Health on Electroconvulsive Therapy. Albany, NY: American Orthopsychiatric Association.

Baddeley, A. D. \& Hitch, G. J. (1974) Working memory. In Recent Advances in Learning and Motivation (vol. 8) (ed. G. A. Bower), pp. 47-90. New York: Academic Press.

Bender, L. (1938) A Visual Motor Gestalt Test and Its Clinical Use. Research Monographs 3. New York: American Orthopsychiatric Association.

Bolwig, T. G., Hertz, M. M. \& Paulson, O. B. (1977) The permeability of the blood-brain barrier during electrically induced seizures in man. European Journal of Clinical Investigation, 7, 87-93.

Broadbent, D. E., Cooper, P. F., FitzGerald, P., et al (1982) The cognitive failures questionnaire (CFQ) and its correlates. British Journal of Clinical Psychology, 21, 1-16.

Brown, L., Sherbenou, L. J. \& Johnsen, S. K. (1982) Test of NonVerbal Intelligence. Austin, TX: Pro-Ed.

Calev, A. (1994) Neuropsychology and ECT: past and future research trends. Psychopharmacology Bulletin, 30, 461-464.

Calev, A., Gaudino, E. A., Squires, N. K., et al (1995) ECT and non-memory cognition: a review. British Journal of Clinical Psychology, 34, 505-515.

Chamberlin, E. \& Tsai, G. E. (1998) A glutamatergic model of ECTinduced memory dysfunction. Harvard Review of Psychiatry, 5, 307-317.

Coleman, E. Z., Sackeim, H. A., Prudic, J., et al (1996) Subjective memory complaints prior to and following electroconvulsive therapy. Biological Psychiatry, 39, 346-356.

Cott, J. (2005) On the Sea of Memory. New York: Random House.

Cronholm, B. \& Ottoson, J.-O. (1963) The experience of memory function after electroconvulsive therapy. British Journal of Psychiatry, 109, 251-258.

Daneman, M. \& Green, I. (1986) Individual differences in comprehending and producing words in context. Journal of Memory and Language, 25, 1-18.

de Filippis, N. A., McCampbell, E. \& Rogers, P. (1979) Development of a booklet form of the Category Test: normative and validatory data. Journal of Clinical Neuropsychology, 1, 339-342.

Diehl, D. J., Keshavan, M. S., Kanal, E., et al (1994) Post-ECT increases in T2 relaxation times and their relationship to cognitive side effects: a pilot study. Psychiatry Research, 54, 177-184.

Donahue, A. (2000) Electroconvulsive therapy and memory loss: a personal journey. Journal of ECT, 16, 133-143.

ECT Accreditation Service (2005) Standards for the Administration of ECT (3rd edn). London: Royal College of Psychiatrists' Research Unit.

ECT Anonymous (UK) (1999) Questionnaire results: March 1999. Riddlesden, Keighsley: ECT Anonymous. 
Food and Drug Administration (1982) Docket \#82P-0316, Electroconvulsive Therapy Device. Rockville, MD: FDA.

Folstein, M. F., Folstein, S. E. \& McHugh, P. R. (1975) "MiniMental State": a practical method for grading the cognitive status of patients for the clinician. Journal of Psychiatric Research, 12, 189-198.

Freeman, C. P., Weeks, D. \& Kendell, R. E. (1980) ECT II: Patients who complain. British Journal of Psychiatry, 137, 8-16.

Goldstein, S. G., Filskov, S. B., Weaver, L. A., et al (1977) Neuropsychological effects of electroconvulsive therapy. Journal of Clinical Psychology, 33, 798-806.

Greenhalgh, J., Knight, C., Hind, D., et al (2005) Clinical and cost-effectiveness of electroconvulsive therapy for depressive illness, schizophrenia, catatonia, and mania: systematic reviews and economic modeling studies. Health Technology Assessment, 9, 1-170.

Heaton, R. K. (1981) Wisconsin Card Sorting Test (WCST). Odessa, FL: Psychological Assessment Resources.

Janis, I. L. (1950) Psychologic effects of electric convulsive treatments (I. Post-treatment amnesias). Journal of Nervous and Mental Disease, 111, 359-381.

Khan, A., Mirolo, M. H., Mirolo, H. A., et al (1993) Can ECTinduced cognitive effects be altered pharmacologically? Progress in Neuropsychopharmacology and Biological Psychiatry, 17, 861-873.

Mattes, J. A., Pettinati, H. M., Stephens, S., et al (1990) A placebocontrolled induced memory impairment. Biological Psychiatry, 27, 289-303.

McCall, W. V., Dunn, A. \& Rosenquist, P. R. (2004) Quality of life and electroconvulsive therapy. British Journal of Psychiatry, 185, 405-409.

National Institute for Clinical Excellence (2003) Guidance on the Use of Electroconvulsive Therapy (Technology Appraisal 59, April). London: NICE. http://www.nice.org.uk/pdf/ 59ectfullguidance.pdf

National Institutes of Health (1985) Electroconvulsive therapy. NIH Consensus Statement Online. June 10-12 [cited year month day]; 5 (11), 1-23. Bethesda, MD: NIH. http:/ / consensus.nih. gov/1985/1985ElectroconvulsiveTherapy051html.htm

Neylan, T. C., Canick, J. D., Hall, S. E., et al (2001) Cortisol levels predict cognitive impairment induced by electroconvulsive therapy. Biological Psychiatry, 50, 331-336.

Pedler, M. (2001) Shock Treatment: A Survey of People's Experience of Electroconvulsive Therapy (ECT). London: Mind.

Pettinati, H. M. \& Rosenberg, J. (1984) Memory self-rating before and after electroconvulsive therapy: depression-versus ECT induced. Biological Psychiatry, 19, 539-548.

Philpot, M., Collins, C., Trivedi, P., et al (2004) Eliciting users' views of ECT in two mental health trusts with a user-designed questionnaire. Journal of Mental Health, 13, 403- 413.

Rami-Gonzalez, L., Bernardo, M., Boget, T., et al (2001) Subtypes of memory dysfunction associated with electroconvulsive therapy. Characteristics and neurobiological bases involved. Journal of ECT, 17, 129-135.

Rami-Gonzalez, L., Salamero, M., Boget, T., et al (2003) Pattern of cognitive dysfunction in depressive patients during maintenance electroconvulsive therapy. Psychological Medicine, 33, 345-350.

Reitan, R. M. \& Wolfson, D. (1993) The Halstead-Reitan Neuropsychological Test Battery: Theory and Clinical Application (2nd edn). Tucson, AZ: Neuropsychology Press.

Rey, A. (1964) L'examen clinique en psychologie. Paris: Presses Universitaires de France.

Rose, D., Fleischmann, P., Wykes, T., et al (2003) Patients' perspectives on electroconvulsive therapy: systematic review. BMJ, 326, 1363-1367.

Rose, D., Wykes, T., Bindman, J., et al (2005) Information, consent and perceived coercion: patients' perspectives on electroconvulsive therapy. British Journal of Psychiatry, 186, 54-59.

Royal College of Psychiatrists (2005) The ECT Handbook (2nd edn) (Council Report CR128). London: Royal College of Psychiatrists.

Sackeim, H. A. (1986) Acute cognitive side effects of ECT. Psychopharmacology Bulletin, 22, 482-484.

Sackeim, H. A. (2000) Memory loss and ECT: from polarization to reconciliation. Journal of ECT, 16, 87-96.
Sackeim, H. A., Devanand, D. P. \& Prudic, J. (1993) Effects of stimulus intensity and electrode placement on the efficacy and cognitive effects of electroconvulsive therapy. New England Journal of Medicine, 328, 839-846.

Sackeim, H. A., Prudic, J. \& Devanand, D. P. (2000) A prospective, randomized double-blind comparison of bilateral and right unilateral ECT at different stimulus intensities. Archives of General Psychiatry, 57, 425- 434.

Schulze-Rauschenbach, S. C., Harms, U., Schlaepfer, T. E., et al (2005) Distinctive neurocognitive effects of repetitive transcranial magnetic stimulation and electroconvulsive therapy in major depression. British Journal of Psychiatry, 186, 410-416.

Scott, A. I. F. (2005) College guidelines on electroconvulsive therapy: an update for prescribers. Advances in Psychiatric Treatment, 11, 150-156.

Service User Research Enterprise (2002) Review of Consumers' Perspectives on Electroconvulsive Therapy. London: Institute of Psychiatry.

Sivan, A. B. (1992) Benton Visual Retention Test (5th edn). San Antonio, TX: Psychological Corporation.

Squire, L. R. \& Chace, P. M. (1975) Memory functions six to nine months after electroconvulsive therapy. Archives of General Psychiatry, 32, 1557-1564.

Squire, L. R. \& Slater, P. C. (1983) Electroconvulsive therapy and complaints of memory dysfunction: a prospective three-year follow-up study. British Journal of Psychiatry, 142, 1-8.

Squire, L. R. \& Zouzounis, J. A. (1988) Self-ratings of memory dysfunction: different findings in depression and amnesia. Journal of Clinical and Experimental Neuropsychology, 10, 727-738.

Squire, L. R., Wetzel, C. D. \& Slater, P. C. (1979) Memory complaints after electroconvulsive therapy: assessment with a new self-rating instrument. Biological Psychiatry, 14, 791-801.

Stoudemire, A., Hill, C. D., Morris, R., et al (1993) Long-term affective and cognitive outcomes in depressed older adults. American Journal of Psychiatry, 148, 1336-1340.

Taylor, J. R., Kuhlengel, B. G. \& Dean, R. S. (1985) ECT, blood pressure changes and neuropsychological deficits. British Journal of Psychiatry, 147, 36-38.

United Kingdom Advocacy Network (1996) ECT Survey: The National Experience. Sheffield: UKAN.

UK ECT Review Group (2003) Efficacy and safety of electroconvulsive therapy in depressive disorders: a systematic review and meta-analysis. Lancet, 361, 799-808.

Wechsler, D. (1997) Wechsler Adult Intelligence Scale III. San Antonio, CA: Psychological Corporation.

Weiner, R. D., Rogers, H. J., Davidson, J. R., et al (1986) Effects of stimulus parameters on cognitive side effects. Annals of the New York Academy of Sciences, 462, 315-325.

Zervas, I. M. \& Jandorf, L. (1993) The Randt memory test in electroconvulsive therapy: relation to illness and treatment parameters. Convulsive Therapy, 9, 28-38.

\section{MCQs}

1 The proportion of patients who will experience permanent retrograde amnesia after ECT is:

a a few outliers

b a small minority

c less than one-third

$\mathrm{d}$ at least one-third

e zero.

2 Permanent retrograde amnesia can best be assessed by:

a the Squire Memory Questionnaire

b the Columbia Autobiographical Memory Inventory

c the MMSE

$\mathrm{d}$ the memory scales of the Wechsler

e the Janis test. 
3 Which of the following statements accurately describe retrograde amnesia?

a studies have proven that it reverses itself within 6 months

b it may be severe, erasing 10 years or more of the patient's life

c it erases only bad memories

d it is limited to dates and details that can be easily relearned

e most patients consider it a small price to pay for ECT's benefits.

4 Difficulty or disability with memory function on an ongoing basis is best described as:

a retrograde amnesia

b anterograde amnesia

c depression

d memory disability

e memory loss

5 Tests that may be useful in assessing non-memory cognition include:

a Wisconsin Card Sort Test

$\mathrm{b}$ paired associates

c the MMSE

d the Benton Visual Retention Test

e the Speaking Span Test.

\title{
MCQ answers
}

$\begin{array}{llllllll}1 & & 2 & & 3 & & & 5 \\ \text { a F } & \text { a F } & \text { a F } & \text { a F } & \text { a T } \\ \text { b F } & \text { b F } & \text { b T } & \text { b T } & \text { b F } \\ \text { c F } & \text { c F } & \text { c F } & \text { c F } & \text { c F } \\ \text { d T } & \text { d F } & \text { d F } & \text { d T } & \text { d T } \\ \text { e F } & \text { e T } & \text { e F } & \text { e F } & \text { e T }\end{array}$

\section{What I would say to a patient who asked me about this article}

\author{
INVITED COMMENTARY ON: MEMORY AND COGNITIVE EFFECTS OF ECT
}

\section{Allan Scott}

Abstract Electroconvulsive therapy (ECT) remains an important treatment option for severe depressive illness, but it can have side-effects, including permanent gaps in memory. Where minimising the intellectual sideeffects of treatment has priority, then treatment to only one side of the head (unilateral ECT) is preferable; where the speed of clinical improvement is paramount, then bilateral ECT may be preferred. The choice of how ECT is administered should, where possible, be part of the process of informed consent.

There are strong, and sometimes conflicting, views about electroconvulsive treatment (ECT). It was for this very reason that the Department of Health commissioned reviews to summarise what is known about the benefits and side-effects of the procedure The review of patients' experiences after ECT (Rose et al,2003) confirmed that permanent gaps in memory, what Robertson \& Pryor (2006, this issue) refer to as permanent amnesia, is a recognised and common side-effect after treatment. The review of clinical trials (UK ECT Review Group, 2003) found that the intellectual side-effects of ECT reflected mostly problems with memory, in particular amnesia. The gaps in memory were not restricted to personal autobiographical events. Few trials investigated the possibility of longer-term intellectual impairments, but those that did suggested that this was not a substantial problem (Carney \& Geddes, 2003). 